\title{
List of Charts
}

Chart 1. Comparison of Thai and Western tuning systems . . . . . . . . . . . 33

Chart 2. Thai pitch levels and pentatonic modes . . . . . . . . . . . . . . 45

Chart 3. Ching patterns . . . . . . . . . . . . . . . . . . . . . . . . 48

Chart 4. Ching patterns . . . . . . . . . . . . . . . . . . . . . . 48

Chart 5. Ching patterns . . . . . . . . . . . . . . . . . . . . . . . . 49

Chart 6. Percentage of occurrences of pitches as a finalis . . . . . . . . . . . 116

Chart 7. Classification of finalis pitches according to pitch levels . . . . . . . . 116

Chart 8. "Khāek Sai" (Thao), pitch outline . . . . . . . . . . . . . . . . 121

Chart 9. "Tuang Phra Thāt" (Thao), Section 1, pitch outline . . . . . . . . . 131

Chart 10. "Tuang Phra Thāt" (Thao), Section 2, pitch outline . . . . . . . . . 133

Chart 11. "Lāo Sīang Thian" (Thao), pitch outline . . . . . . . . . . . . . . 136

Chart 12. "Lao Khruan" (2 chan), pitch outline . . . . . . . . . . . . . . . 139

Chart 13. "Lāo Chĩang Tat Sọì" (2 chan), pitch outline . . . . . . . . . . . . 140

Chart 14. "Nok Khao Khamae" (Thao), pitch outline _. . . . . . . . . . . . 141

Chart 15. "Khamāe Thom" (3 chan) . . . . . . . . . . . . . . . . . . . 141

Chart 16. "Lìlā K(r)athum" (3 chan) . . . . . . . . . . . . . . . . . . . 146

Chart 17. "Sām Mai Nai" (Thao) . . . . . . . . . . . . . . . . . . . . 148

Chart 18. Pitch-exchange process for metabole . . . . . . . . . . . . . . . 150

Chart 19. "Hong Thōng" (Thao), pitch outline . . . . . . . . . . . . . . . 152

Chart 20. "Khāek Thọ̄n Sāi Bua" (2 chan), pitch outline . . . . . . . . . . . 153

Chart 21. "Phrayã Khruan" (3 chan), pitch outline . . . . . . . . . . . . . 155

Chart 22. "Ȳ̧ng NGit" (Thai), pitch outline . . . . . . . . . . . . . . . . 158

Chart 23. "Phat Chā" (3 chan), pitch outline . . . . . . . . . . . . . . . . 158

Chart 24. "Dōk Mai Sai" (3 chan), pitch outline . . . . . . . . . . . . . . . . 159

Chart 25. "Khāè M̄̄n Bāng Chāng" (3 chan), pitch outline . . . . . . . . . . 164

Chart 26. "Sōi Mayurā" (Thao) . . . . . . . . . . . . . . . . . . . . . 165

Chart 27. "Rasam Rasāi" (3 chan) . . . . . . . . . . . . . . . . . . . . 167

Chart 28. "Chāng Prasān NGā"' (Thao) . . . . . . . . . . . . . . . . . . . . . . . 169

Chart 29. Classification of rựang . . . . . . . . . . . . . . . . . . . . . . . 181

Chart 30. "Lā" (Farewell Song), pitch outline . . . . . . . . . . . . . . . 182

Chart 31. "Nok Khao Khamae" (Thao), pitch outline . . . . . . . . . . . . 188

Chart 32. "Khāek Sai" (Ru̧ūng), pitch outline . . . . . . . . . . . . . . . 189

Chart 33. "Khāek Sai" (Thao), pitch outline . . . . . . . . . . . . . . . . 189

Chart 34. "Ānū" (Ŗَ̧ang), pitch outline . . . . . . . . . . . . . . . . . . 190

Chart 35. "Ānū" (Thao), pitch outline . . . . . . . . . . . . . . . . . . 195

Chart 36. "Khamēn Yai" and "Khamāe Thom" (Rữāng and 3 chan), pitch outline . . . . . . . . . . . . . . . . . . . . . . . 197

Chart 37. "Khāek T⿳̄i Mọ"” (Thao), pitch outline . . . . . . . . . . . . . . 206

Chart 38. "Khamēn Pāk Thō" (Thao), pitch outline . . . . . . . . . . . . . 222 
